\title{
On weak solutions to the 2D Savage-Hutter model of the motion of a gravity driven avalanche flow
}

\author{
Eduard Feireisl * $\quad$ Piotr Gwiazda ${ }^{\dagger} \quad$ Agnieszka Świerczewska-Gwiazda ${ }^{\ddagger}$
}

\author{
Institute of Mathematics of the Academy of Sciences of the Czech Republic \\ Žitná 25, 11567 Praha 1, Czech Republic \\ Institute of Applied Mathematics and Mechanics, University of Warsaw \\ Banacha 2, 02-097 Warszawa, Poland
}

\begin{abstract}
We consider the Savage-Hutter system consisting of two-dimensional depth-integrated shallow water equations for the incompressible fluid with the Coulomb-type friction term. Using the method of convex integration we show that the associated initial-value problem possesses infinitely many weak solutions for any finite-energy initial data. On the other hand, the problem enjoys the weak-strong uniqueness property provided the system of equations is supplemented with the energy inequality.
\end{abstract}

Key words: Savage-Hutter system, avalanche flow, weak solution, convex integration

\section{Introduction}

The theory for gravity driven avalanche flows is qualitatively similar to that of compressible fluid dynamics. We consider a relatively simple Savage-Hutter model based on the assumption that the

${ }^{*}$ The research of E.F. leading to these results has received funding from the European Research Council under the European Union's Seventh Framework Programme (FP7/2007-2013)/ ERC Grant Agreement 320078

${ }^{\dagger} \mathrm{P}$. G. is a coordinator of the International Ph.D. Projects Programme of the Foundation for Polish Science operated within the Innovative Economy Operational Programme 2007-2013 (Ph.D. Programme: Mathematical Methods in Natural Sciences)

¥The research of A.Ś.-G. has received funding from the National Science Centre, DEC-2012/05/E/ST1/02218 
material is incompressible, with an isotropic pressure distribution through its depth, and a Coulomb sliding friction, see Gray et al. [14] and [17] for a comprehensive overview. The time evolution of the flow height $h=h(t, x)$ and depth-averaged velocity $\mathbf{u}=\mathbf{u}(t, x)$ is described through a system of balance laws - the Savage-Hutter system:

$$
\begin{gathered}
\partial_{t} h+\operatorname{div}_{x}(h \mathbf{u})=0 \\
\partial_{t}(h \mathbf{u})+\operatorname{div}_{x}(h \mathbf{u} \otimes \mathbf{u})+\nabla_{x}\left(a h^{2}\right)=h\left(-\gamma \frac{\mathbf{u}}{|\mathbf{u}|}+\mathbf{f}\right)
\end{gathered}
$$

where $|\cdot|$ is an Euclidean metric, $a \geq 0, \gamma \geq 0$, and $\mathbf{f}$ are given (smooth) functions of spatial coordinate $x \in \Omega \subset R^{2}$, see Gray and Cui [13], Zahibo et al. [23]. For the sake of simplicity, we restrict ourselves to the periodic boundary conditions supposing accordingly that $\Omega$ is the "flat" torus

$$
\Omega=\left(\left.[0,1]\right|_{\{0,1\}}\right)^{2} .
$$

The system (1.1), (1.2) is supplemented with the initial conditions

$$
h(0, \cdot)=h_{0}, \mathbf{u}(0, \cdot)=\mathbf{u}_{0}
$$

The term $\frac{\mathbf{u}}{|\mathbf{u}|}$ has to be understood as a multi-valued mapping, which for non-zero velocities takes the value $\frac{\mathbf{u}}{|\mathbf{u}|}$, whereas for $\mathbf{u}=0$ takes the values in the whole closed unit ball. For the physical justification for such formulation with a simple argument on one-dimensional steady solutions we refer to [15].

In the absence of the driving force on the right-hand side of (1.2), the Savage-Hutter system coincides with barotropic Euler system describing the motion of a compressible inviscid fluid. As observed Gray and Cui [13], the solutions of the Savage-Hutter system develop shock waves and other singularities characteristic for hyperbolic system of conservation laws. Accordingly, any mathematical theory based on the classical concept of (smooth) solutions fails as soon as we are interested in globalin-time solutions to the system (1.1), (1.2), and/or in solutions emanating from singular initial data. Hence in analogue to the development of the theory for Euler equations, the problem of existence of weak solutions has so far remained open. The issue of measure-valued solutions for the twodimensional model considered here was studied in [16]. Again, similar as for Euler flows, the author follows the concept of generalization by DiPerna and Majda [9] to capture both oscillations as well as concentration effects. The existence of entropy weak solution to the corresponding problem in one-dimensional setting was shown in [15]. Various modifications of the model to more complex topographies were considered in [1, 2, 12], see also [18, 19] for computational results. 
In this paper, we consider the weak solutions to the problem (1.1 1.4) determined by means of a family of integral identities:

$$
\int_{0}^{T} \int_{\Omega}\left(h \partial_{t} \varphi+h \mathbf{u} \cdot \nabla_{x} \varphi\right) \mathrm{d} x \mathrm{~d} t=-\int_{\Omega} h_{0} \varphi(0, \cdot) \mathrm{d} x \mathrm{~d} t
$$

for any $\varphi \in C^{1}([0, T) \times \Omega)$;

$$
\begin{aligned}
& \int_{0}^{T} \int_{\Omega}\left(h \mathbf{u} \cdot \partial_{t} \Phi+h \mathbf{u} \otimes \mathbf{u}: \nabla_{x} \Phi+a h^{2} \operatorname{div}_{x} \Phi\right) \mathrm{d} x \mathrm{~d} t \\
& =\int_{0}^{T} \int_{\Omega} h(\gamma \mathbf{B}-\mathbf{f}) \cdot \Phi \mathrm{d} x \mathrm{~d} t-\int_{\Omega} h_{0} \mathbf{u}_{0} \cdot \Phi(0, \cdot) \mathrm{d} x
\end{aligned}
$$

for any $\Phi \in C^{1}\left([0, T) \times \Omega ; R^{2}\right)$, where

$$
\mathbf{B}=\mathbf{B}_{\mathbf{u}}(t, x)=\left\{\begin{array}{l}
\frac{\mathbf{u}(t, x)}{|\mathbf{u}(t, x)|} \text { if } \mathbf{u}(t, x) \neq 0, \\
\in \overline{B_{1}(0)} \text { if } \mathbf{u}(t, x)=0,
\end{array}\right.
$$

where $B_{1}(0)$ is the unit ball in $\mathbb{R}^{2}$ with respect to the Euclidean metric. Hence speaking about weak solutions we in fact mean a triplet $\left[h, \mathbf{u}, \mathbf{B}_{\mathbf{u}}\right]$, whereas $\mathbf{B}_{\mathbf{u}}$ is the selection from the multi-valued graph.

Using the method of convex integration, recently adapted to the incompressible Euler system by De Lellis and Székelyhidi [7, and Chiodaroli [3], we show that the Savage-Hutter system is always solvable but not well posed in the class of weak solutions. More specifically, we show that the problem (1.1 1.4) admits infinitely many weak solutions on a given time interval $(0, T)$ and for any sufficiently smooth initial data, see Section 2. The method of convex integration is used to construct large sets of weak solutions. Starting from the seminal results of Scheffer [20] an Shnirelman [21] on existence of compactly supported $2 D$ weak solutions to the Euler equations and the previously mentioned result of De Lellis and Székelyhidi [7]. The theory was extensively developed for constructng weak solutions with bounded energy by Wiedemann [22] and in a nontrivial way extended for compressible Euler in the so-called variable coefficient variant of the approach [3, 5] and recently to more complex systems such as equations of a compressible heat conducting gas [4], Euler-Korteweg-Poisson system [10].

Apparently, the approach of convex integration allows to avoid the difficulties arising from the multi-valued formulation of the friction term. In case of measure-valued solutions which were generated by the approximate sequences of corresponding viscous problems one could not exclude the case of $\mathbf{u}=0$, cf. Gwiazda [16] and also the same difficulty in $1 D$ case [15]. In the current framework we choose $h$ and the energy $E$ and the consequent steps lead to finding the momentum. Although the friction term was nonlinear in terms of $\mathbf{u}$, the situation turns out to be significantly better as we express the friction term as a product of a scalar function only of $h$ and $E$ and a function of $h$ and $h u$ which is linear. The linearity then obviously provides weak continuity. And what is the 
most essential, postulating $h>0$ and the energy being sufficiently large yields that the velocity $\mathbf{u}$ is non-zero, and hence we are away from the set where the friction term is multi-valued.

Next, in Section 3, we augment the weak formulation (1.5) 1.7) by the energy inequality. Moreover, using the relative energy method introduced by Dafermos [6] and developed in [11], we show the weakstrong uniqueness principle: A weak solution satisfying the energy inequality necessarily coincides with the strong solution emanating from the same initial data as long as the latter exists. The paper is concluded by some remarks concerning the implications of the convex integration technique on the well-posedness problem considered in the class of finite energy weak solutions, see Section 4 .

We conclude the introduction with a remark that the current studies are a negative result for numerical simulation conducted for this system as the analysis shows that solutions with a sufficiently large energy exist but are non-unique. Nevertheless, this does not exclude there might exist solutions with a small energy, which are unique.

\section{Infinitely many weak solutions}

We start with a brief description of the procedure of constructing the solutions. An essential tool is the Baire category method arising from the theory of differential inclusions. Note however that instead of the abstract arguments one could provide the constructive, however essentially longer proof by adding oscillatory perturbations. Similarly to [4], we will start with regular initial data

$$
h_{0} \in C^{2}(\Omega), \mathbf{u}_{0} \in C^{2}\left(\Omega ; R^{2}\right), h_{0}>0 \text { in } \Omega .
$$

and reformulate the problem in different variables in order to obtain the system corresponding to an incompressible Euler system. The regularity of data appears to be important in the oscillatory lemma, which the same as in case of compressible Euler flow is formulated in the variable coefficients form with the coefficients generated by the data. For the new system we will construct a family of subsolutions. Indeed, we rewrite it as a linear system coupled with a nonlinear constraint. Firstly, completing the space of subsolutions in appropriate topology we consider a family of functionals, which turn out to be lower-semicontinuous. In the next step we will conclude that as the limit object is a pointwise limit of continuous functions, namely it is Baire-1 function, then the set of continuity points is infinite. To show that the points where the functional vanishes are solutions to the system we will use an oscillatory lemma, namely Lemma 2.1.

In the first step, using the standard Helmholtz decomposition, we may write

$$
h_{0} \mathbf{u}_{0}=\mathbf{v}_{0}+\mathbf{V}_{0}+\nabla_{x} \Psi_{0}, \text { where } \operatorname{div}_{x} \mathbf{v}_{0}=0, \int_{\Omega} \Psi_{0} \mathrm{~d} x=0, \int_{\Omega} \mathbf{v}_{0} \mathrm{~d} x=0, \mathbf{V}_{0} \in R^{2}
$$

The main result proved in the section reads: 
Theorem 2.1 Let $T>0$ and the initial data $h_{0}, \mathbf{u}_{0}$ satisfying (2.1) be given. Suppose that $a, \gamma \in C^{2}(\Omega), a, \gamma \geq 0, \mathbf{f} \in C^{1}\left([0, T] \times \Omega ; R^{2}\right)$.

Then the problem $(1.1-1.4)$ admits infinitely many weak solutions in $(0, T) \times \Omega$. The weak solutions belong to the class

$$
\begin{gathered}
h, \partial_{t} h, \nabla_{x} h \in C^{1}([0, T] \times \Omega), \\
\mathbf{u} \in C_{\text {weak }}\left([0, T] ; L^{2}\left(\Omega ; R^{2}\right)\right) \cap L^{\infty}\left((0, T) \times \Omega ; R^{2}\right), \operatorname{div}_{x} \mathbf{u} \in C([0, T] \times \Omega), \\
\mathbf{B} \in L^{\infty}\left((0, T) \times \Omega ; R^{2}\right) .
\end{gathered}
$$

Above, by $C_{\text {weak }}\left([0, T] ; L^{2}\left(\Omega ; R^{2}\right)\right)$ we mean the space of functions continuous in time with respect to the weak topology of $L^{2}\left(\Omega ; R^{2}\right)$. The remaining part of this section is devoted to the proof of Theorem 2.1.

\subsection{Convex integration ansatz}

Similarly to the decomposition (2.2), we look for solutions in the form

$$
h \mathbf{u}=\mathbf{v}+\mathbf{V}+\nabla_{x} \Psi, \text { where } \operatorname{div}_{x} \mathbf{v}=0, \int_{\Omega} \Psi(t, \cdot) \mathrm{d} x=0, \int_{\Omega} \mathbf{v}(t, \cdot) \mathrm{d} x=0, \mathbf{V}=\mathbf{V}(t) \in R^{2} .
$$

Thus the continuity equation (1.1) reads

$$
\partial_{t} h+\Delta_{x} \Psi=0 \text { in }(0, T) \times \Omega, h(0, \cdot)=h_{0}, \Psi(0, \cdot)=\Psi_{0} .
$$

Now, we can choose $h=h(t, x) \in C^{2}([0, T] \times \Omega)$ in such a way that

$$
h(0, \cdot)=h_{0}, \partial_{t} h(0, \cdot)=-\Delta_{x} \Psi_{0}, h(t, \cdot)>0, \int_{\Omega} h(t, \cdot) \mathrm{d} x=\int_{\Omega} h_{0} \mathrm{~d} x \text { for all } t \in[0, T],
$$

and compute

$$
-\Delta_{x} \Psi(t, \cdot)=\partial_{t} h(t, \cdot), \int_{\Omega} \Psi(t, \cdot) \mathrm{d} x=0 .
$$

Consequently, the original problem (1.1 1.4) reduces to finding the functions $\mathbf{v}, \mathbf{V}$ satisfying (weakly)

$$
\partial_{t} \mathbf{v}+\partial_{t} \mathbf{V}+\operatorname{div}_{x}\left(\frac{\left(\mathbf{v}+\mathbf{V}+\nabla_{x} \Psi\right) \otimes\left(\mathbf{v}+\mathbf{V}+\nabla_{x} \Psi\right)}{h}+\left(a h^{2}+\partial_{t} \Psi\right) \mathbb{I}\right)
$$




$$
\begin{gathered}
=h\left(-\gamma \frac{\mathbf{v}+\mathbf{V}+\nabla_{x} \Psi}{\left|\mathbf{v}+\mathbf{V}+\nabla_{x} \Psi\right|}+\mathbf{f}\right), \\
\operatorname{div}_{x} \mathbf{v}=0, \int_{\Omega} \mathbf{v}(t, \cdot) \mathrm{d} x=0 \\
\mathbf{v}(0, \cdot)=\mathbf{v}_{0}, \mathbf{V}(0)=\mathbf{V}_{0} .
\end{gathered}
$$

\section{$2.2 \quad$ Kinetic energy}

We denote

$$
E=\frac{1}{2} \frac{\left|\mathbf{v}+\mathbf{V}+\nabla_{x} \Psi\right|^{2}}{h}
$$

the kinetic energy associated with the Savage-Hutter system. Analogously, we rewrite (2.4) in the form

$$
\begin{aligned}
\partial_{t} \mathbf{v}+ & \partial_{t} \mathbf{V}+\operatorname{div}_{x}\left(\frac{\left(\mathbf{v}+\mathbf{V}+\nabla_{x} \Psi\right) \otimes\left(\mathbf{v}+\mathbf{V}+\nabla_{x} \Psi\right)}{h}-\frac{1}{2} \frac{\left|\mathbf{v}+\mathbf{V}+\nabla_{x} \Psi\right|^{2}}{h} \mathbb{I}\right) \\
& +\nabla_{x}\left(E-\Lambda+a h^{2}+\partial_{t} \Psi\right)=-\gamma\left(\frac{h}{2 E}\right)^{1 / 2}\left(\mathbf{v}+\mathbf{V}+\nabla_{x} \Psi\right)+h \mathbf{f},
\end{aligned}
$$

where $\Lambda=\Lambda(t)$ is a spatially homogeneous function to be determined below. Finally, for

$$
E=\Lambda-a h^{2}-\partial_{t} \Psi
$$

equation (2.8) reduces to

$$
\begin{gathered}
\partial_{t} \mathbf{v}+\partial_{t} \mathbf{V}+\operatorname{div}_{x}\left(\frac{\left(\mathbf{v}+\mathbf{V}+\nabla_{x} \Psi\right) \otimes\left(\mathbf{v}+\mathbf{V}+\nabla_{x} \Psi\right)}{h}-\frac{1}{2} \frac{\left|\mathbf{v}+\mathbf{V}+\nabla_{x} \Psi\right|^{2}}{h} \mathbb{I}\right) \\
=-\gamma\left(\frac{h}{2 E}\right)^{1 / 2}\left(\mathbf{v}+\mathbf{V}+\nabla_{x} \Psi\right)+h \mathbf{f} .
\end{gathered}
$$

\subsection{Determining V}

The spatially homogeneous function $\mathbf{V}$ is determined as the unique solution of the ordinary differential equation

$$
\partial_{t} \mathbf{V}-\left[\frac{1}{|\Omega|} \int_{\Omega} \gamma\left(\frac{h}{2 E}\right)^{1 / 2} \mathrm{~d} x\right] \mathbf{V}=\frac{1}{|\Omega|} \int_{\Omega}\left[\gamma\left(\frac{h}{2 E}\right)^{1 / 2}\left(\mathbf{v}+\nabla_{x} \Psi\right)+h \mathbf{f}\right] \mathrm{d} x, \mathbf{V}(0)=\mathbf{V}_{0}
$$

Note that $\mathbf{V}=\mathbf{V}[\mathbf{v}]$ depends on $\mathbf{v}$ and also on the function $\Lambda$ in (2.9). 
With such a choice of $\mathbf{V}$, equation (2.10) reads

$$
\begin{gathered}
\partial_{t} \mathbf{v}+\operatorname{div}_{x}\left(\frac{\left(\mathbf{v}+\mathbf{V}[\mathbf{v}]+\nabla_{x} \Psi\right) \otimes\left(\mathbf{v}+\mathbf{V}[\mathbf{v}]+\nabla_{x} \Psi\right)}{h}-\frac{1}{2} \frac{\left|\mathbf{v}+\mathbf{V}[\mathbf{v}]+\nabla_{x} \Psi\right|^{2}}{h} \mathbb{I}\right) \\
=-\gamma\left(\frac{h}{2 E}\right)^{1 / 2}\left(\mathbf{v}+\mathbf{V}[\mathbf{v}]+\nabla_{x} \Psi\right)+\frac{1}{|\Omega|} \int_{\Omega} \gamma\left(\frac{h}{2 E}\right)^{1 / 2}\left(\mathbf{v}+\mathbf{V}[\mathbf{v}]+\nabla_{x} \Psi\right) \mathrm{d} x+h \mathbf{f}-\frac{1}{|\Omega|} \int_{\Omega} h \mathbf{f} \mathrm{d} x .12
\end{gathered}
$$

Finally, we find a tensor $\mathbb{M}=\mathbb{M}[\mathbf{v}]$ such that $\mathbb{M}(t, x) \in R_{\mathrm{sym}, 0}^{2 \times 2}$, where $R_{\mathrm{sym}, 0}^{2 \times 2}$ is the set of $2 \times 2$ symmetric traceless matrices, for any $t, x$, and

$$
\begin{gathered}
\operatorname{div}_{x} \mathbb{M} \\
=-\gamma\left(\frac{h}{2 E}\right)^{1 / 2}\left(\mathbf{v}+\mathbf{V}[\mathbf{v}]+\nabla_{x} \Psi\right)+\frac{1}{|\Omega|} \int_{\Omega} \gamma\left(\frac{h}{2 E}\right)^{1 / 2}\left(\mathbf{v}+\mathbf{V}[\mathbf{v}]+\nabla_{x} \Psi\right) \mathrm{d} x+h \mathbf{f}-\frac{1}{|\Omega|} \int_{\Omega} h \mathbf{f} \mathrm{d} x \\
\int_{\Omega} \mathbb{M}(t, \cdot) \mathrm{d} x=0 \text { for any } t \in[0, T]
\end{gathered}
$$

We can take

$$
\mathbb{M}=\nabla_{x} \mathbf{m}+\nabla_{x}^{t} \mathbf{m}-\operatorname{div}_{x} \mathbf{m} \mathbb{I},
$$

where $\mathbf{m}$ is the (unique) solution of the elliptic equation

$$
\begin{gathered}
\operatorname{div}_{x}\left(\nabla_{x} \mathbf{m}+\nabla_{x}^{t} \mathbf{m}-\operatorname{div}_{x} \mathbf{m} \mathbb{I}\right) \\
=-\gamma\left(\frac{h}{2 E}\right)^{1 / 2}\left(\mathbf{v}+\mathbf{V}[\mathbf{v}]+\nabla_{x} \Psi\right)+\frac{1}{|\Omega|} \int_{\Omega} \gamma\left(\frac{h}{2 E}\right)^{1 / 2}\left(\mathbf{v}+\mathbf{V}[\mathbf{v}]+\nabla_{x} \Psi\right) \mathrm{d} x+h \mathbf{f}-\frac{1}{|\Omega|} \int_{\Omega} h \mathbf{f} \mathrm{d} x \\
\int_{\Omega} \mathbf{m ~ d} x=0 .
\end{gathered}
$$

Indeed Desvilletes and Villani showed a variant of Korn's inequality [8, Section IV.I, Proposition 11]

$$
\left\|\nabla_{x} \mathbf{m}+\nabla_{x}^{t} \mathbf{m}-\operatorname{div}_{x} \mathbf{m} \mathbb{I}\right\|_{L^{2}\left(\Omega ; R^{2 \times 2}\right)} \geq \frac{1}{2}\left\|\nabla_{x} \mathbf{m}\right\|_{L^{2}\left(\Omega ; R^{2 \times 2}\right)},
$$

in particular, the problem (2.14), (2.15) admits a unique solution.

Summarizing, we write equation (2.12) in a concise form

$$
\partial_{t} \mathbf{v}+\operatorname{div}_{x}\left(\frac{\left(\mathbf{v}+\mathbf{V}[\mathbf{v}]+\nabla_{x} \Psi\right) \otimes\left(\mathbf{v}+\mathbf{V}[\mathbf{v}]+\nabla_{x} \Psi\right)}{h}-\frac{1}{2} \frac{\left|\mathbf{v}+\mathbf{V}[\mathbf{v}]+\nabla_{x} \Psi\right|^{2}}{h} \mathbb{I}-\mathbb{M}[\mathbf{v}]\right)=0
$$

where $\mathbf{V}, \mathbb{M}$ are determined by means of (2.11), (2.13), respectively. 


\subsection{Application of the method of convex integration}

In order to recast our problem in terms of the method proposed by De Lellis and Székelyhidi [7], we need to determine

- a (topological) space of subsolutions $X_{0}$;

- a lower semi-continuous functional $I: \bar{X}_{0} \rightarrow R$ such that the points of continuity of $I$ coincide with the solution set of our problem.

\subsubsection{Subsolutions}

Let $\lambda_{\max }[\mathbb{A}]$ denote the maximal eigenvalue of a symmetric matrix $\mathbb{A}$. Motivated by [7], we introduce the set

$$
\begin{gathered}
X_{0}=\left\{\mathbf{w} \mid \mathbf{w} \in L^{\infty}\left((0, T) \times \Omega, R^{2}\right) \cap C^{1}\left((0, T) \times \Omega, R^{2}\right) \cap C_{\text {weak }}\left([0, T] ; L^{2}\left(\Omega ; R^{2}\right)\right),\right. \\
\mathbf{w}(0, \cdot)=\mathbf{v}_{0}, \operatorname{div}_{x} \mathbf{w}=0 \text { in }(0, T) \times \Omega, \\
\partial_{t} \mathbf{w}+\operatorname{div}_{x} \mathbb{F}=0 \text { in }(0, T) \times \Omega \text { for some } \mathbb{F} \in C^{1}\left((0, T) \times \Omega ; R_{0, \text { sym }}^{2 \times 2}\right), \\
\lambda_{\max }\left[\frac{\left(\mathbf{w}+\mathbf{V}[\mathbf{w}]+\nabla_{x} \Psi\right) \otimes\left(\mathbf{w}+\mathbf{V}[\mathbf{w}]+\nabla_{x} \Psi\right)}{h}-\mathbb{F}-\mathbb{M}[\mathbf{w}]\right]<E-\delta \\
\operatorname{in~}(0, T) \times \Omega \text { for some } \delta>0\},
\end{gathered}
$$

where $E$ is the kinetic energy introduced in (2.9).

The first observation is that the set $X_{0}$ is non-empty provided

$$
\Lambda(t) \geq \Lambda_{0}>0 \text { in }[0, T]
$$

in (2.9), and $\Lambda_{0}$ is large enough. Here "large enough" means in terms of the initial data, $\mathbf{f}$, and the time $T$. Indeed, taking $\mathbf{w}=\mathbf{v}_{\mathbf{0}}, \mathbb{F}=0$, we have to find $\Lambda_{0}$ such that

$$
\lambda_{\max }\left[\frac{\left(\mathbf{v}_{0}+\mathbf{V}\left[\mathbf{v}_{0}\right]+\nabla_{x} \Psi\right) \otimes\left(\mathbf{v}_{0}+\mathbf{V}\left[\mathbf{v}_{0}\right]+\nabla_{x} \Psi\right)}{h}-\mathbb{M}\left[\mathbf{v}_{0}\right]\right]<E-\delta=\Lambda-a h^{2}-\partial_{t} \Psi-\delta .
$$

Since $\mathbf{V}$ is given by (2.11), it is easy to check that $\mathbf{V}\left[\mathbf{v}_{0}\right]$, together with $\partial_{t} \mathbf{V}\left[\mathbf{v}_{0}\right]$, remain bounded in terms of the data and uniformly for all $\Lambda \geq \Lambda_{0}$. Furthermore, applying the standard elliptic estimates to (2.13), we get

$$
\|\mathbb{M}(t, \cdot)\|_{W^{1, q}\left(\Omega ; R^{2 \times 2}\right)} \leq c(q \text {, data }) \text { for any } 1<q<\infty, t \in[0, T] .
$$

Consequently, we may fix $\Lambda_{0}, \Lambda$ satisfying (2.17), and, finally, the kinetic energy $E$ in (2.8) in such a way that the set of subsolutions $X_{0}$ is non-empty. 


\subsubsection{Uniform bounds}

As shown by De Lellis and Székelyhidi [7], we have

$$
\begin{gathered}
\frac{1}{2} \frac{\left|\mathbf{w}+\mathbf{V}[\mathbf{w}]+\nabla_{x} \Psi\right|^{2}}{h} \\
\leq \lambda_{\max }\left[\frac{\left(\mathbf{w}+\mathbf{V}[\mathbf{w}]+\nabla_{x} \Psi\right) \otimes\left(\mathbf{w}+\mathbf{V}[\mathbf{w}]+\nabla_{x} \Psi\right)}{h}-\mathbb{F}-\mathbb{M}[\mathbf{w}]\right],
\end{gathered}
$$

where the equality holds only if

$$
\mathbb{F}+\mathbb{M}[\mathbf{w}]=\frac{\left(\mathbf{w}+\mathbf{V}[\mathbf{w}]+\nabla_{x} \Psi\right) \otimes\left(\mathbf{w}+\mathbf{V}[\mathbf{w}]+\nabla_{x} \Psi\right)}{h}-\frac{\left|\mathbf{w}+\mathbf{V}[\mathbf{w}]+\nabla_{x} \Psi\right|^{2}}{h} \mathbb{I} .
$$

Since $E$ has been fixed, we may deduce from (2.18) that

$$
|\mathbf{w}+\mathbf{V}[\mathbf{w}]| \leq c(E, T, \text { data }) \text { in }(0, T) \times \Omega,
$$

and, going back to (2.11), we may infer that

$$
|\mathbf{w}|+|\mathbf{V}[\mathbf{w}]|+\left|\partial_{t} \mathbf{V}[\mathbf{w}]\right| \leq c(E, T \text {, data }) \text { in }(0, T) \times \Omega .
$$

Furthermore, (2.14) yields

$$
\|\mathbb{M}[\mathbf{w}](t, \cdot)\|_{W^{1, q}\left(\Omega ; R^{2 \times 2}\right)} \leq c(E, T \text {, data }) \text { in }(0, T) \text { for any } 1 \leq q<\infty .
$$

The set $X_{0}$ is endowed with the topology of the space $C_{\text {weak }}\left([0, T] ; L^{2}\left(\Omega ; R^{2}\right)\right)$. In view of $(2.20)$, such a topology is metrizable on $X_{0}$ and we denote $\bar{X}_{0}$ the completion of $X_{0}$ - a topological metric space. In accordance with (2.21), (2.22), and the compact embedding $W^{1, q} \hookrightarrow \hookrightarrow C(\Omega), q>2$, we obtain

$$
\left.\begin{array}{r}
\mathbf{V}\left[\mathbf{w}_{n}\right] \rightarrow \mathbf{V}[\mathbf{w}] \text { in } C[0, T] \\
\mathbb{M}\left[\mathbf{w}_{n}\right] \rightarrow \mathbb{M}[\mathbf{w}] \text { in } C([0, T] \times \Omega)
\end{array}\right\} \text { whenever } \mathbf{w}_{n} \rightarrow \mathbf{w} \text { in } C_{\text {weak }}\left([0, T] ; L^{2}\left(\Omega ; R^{2}\right)\right)
$$

\subsubsection{Functional $I$ and infinitely many solutions}

Following De Lellis and Székelyhidi [7], we introduce the functional

$$
I[\mathbf{v}]=\int_{0}^{T} \int_{\Omega}\left[\frac{1}{2} \frac{\left|\mathbf{v}+\mathbf{V}[\mathbf{v}]+\nabla_{x} \Psi\right|^{2}}{h}-E\right] \mathrm{d} x \mathrm{~d} t: \bar{X}_{0} \rightarrow R .
$$

In order to proceed, we need the following variant of the oscillatory lemma (cf. De Lellis and Székelyhidi [7, Proposition 3], Chiodaroli [3, Section 6, formula (6.9)]) proved in [10, Lemma 3.1] : 
Lemma 2.1 Let $U \subset R \times R^{N}, N=2,3$ be a bounded open set. Suppose that

$$
\mathbf{g} \in C\left(U ; R^{N}\right), \mathbb{W} \in C\left(U ; R_{\mathrm{sym}, 0}^{N \times N}\right), e, r \in C(U), r>0, e \leq \bar{e} \text { in } U
$$

are given such that

$$
\frac{N}{2} \lambda_{\max }\left[\frac{\mathbf{g} \otimes \mathbf{g}}{r}-\mathbb{W}\right]<e \text { in } U
$$

Then there exist sequences

$$
\mathbf{w}_{n} \in C_{c}^{\infty}\left(U ; R^{N}\right), \mathbb{G}_{n} \in C_{c}^{\infty}\left(U ; R_{\mathrm{sym}, 0}^{N \times N}\right), n=0,1, \ldots
$$

such that

$$
\begin{gathered}
\partial_{t} \mathbf{w}_{n}+\operatorname{div}_{x} \mathbb{G}_{n}=0, \operatorname{div}_{x} \mathbf{w}_{n}=0 \text { in } R^{N}, \\
\frac{N}{2} \lambda_{\max }\left[\frac{\left(\mathbf{g}+\mathbf{w}_{n}\right) \otimes\left(\mathbf{g}+\mathbf{w}_{n}\right)}{r}-\left(\mathbb{W}+\mathbb{G}_{n}\right)\right]<e \text { in } U,
\end{gathered}
$$

and

$$
\mathbf{w}_{n} \rightarrow 0 \text { weakly in } L^{2}\left(U ; R^{N}\right), \liminf _{n \rightarrow \infty} \int_{U} \frac{\left|\mathbf{w}_{n}\right|^{2}}{r} \mathrm{~d} x \mathrm{~d} t \geq c(\bar{e}) \int_{U}\left(e-\frac{1}{2} \frac{|\mathbf{g}|^{2}}{r}\right)^{2} \mathrm{~d} x \mathrm{~d} t .
$$

Remark 2.1 It is important to note that the constant $c(\bar{e})$ in (2.24) is independent of the specific form of the quantities $\mathbf{g}, \mathbb{W}$, e, and $r$.

In view of (2.18), we have

$$
I[\mathbf{w}]<0 \text { for any } \mathbf{w} \in X_{0}
$$

and, as a consequence of (2.23) $, I: \bar{X}_{0} \rightarrow(-\infty, 0]$ is a lower semi-continuous functional with respect to the topology of the space $C_{\text {weak }}\left([0, T] ; L^{2}\left(\Omega ; R^{2}\right)\right)$. Consequently, by virtue of Baires category argument, the set of points of continuity of $I$ in $\bar{X}_{0}$ has infinite cardinality. Our ultimate goal will be to show that

$$
I[\mathbf{v}]=0 \text { whenever } \mathbf{v} \in \bar{X}_{0} \text { is a point of continuity of } I \text { in } \bar{X}_{0} .
$$

In view of (2.9), (2.11), (2.14), and (2.18), (2.19), it is easy to check that $\mathbf{v}$ represents a weak solution of the problem (2.4 2.6), which completes the proof of Theorem 2.1.

To see (2.25), arguing by contradiction, we assume that $\mathbf{v} \in \bar{X}_{0}$ is a point of continuity of $I$ such that

$$
I[\mathbf{v}]<0 .
$$


Since $I$ is continuous at $\mathbf{v}$, there exists a sequence $\left\{\mathbf{v}_{m}\right\}_{m=1}^{\infty} \subset X_{0}$ (and the associated fluxes $\mathbb{F}_{m}$ ) such that

$$
\mathbf{v}_{m} \rightarrow \mathbf{v} \text { in } C_{\text {weak }}\left([0, T] ; L^{2}\left(\Omega ; R^{2}\right)\right), I\left[\mathbf{v}_{m}\right] \rightarrow I[\mathbf{v}] \text { as } m \rightarrow \infty .
$$

As $\left[\mathbf{v}_{m}, \mathbb{F}_{m}\right]$ are subsolutions, we get

$$
\begin{gathered}
\lambda_{\max }\left[\frac{\left(\mathbf{v}_{m}+\mathbf{V}\left[\mathbf{v}_{m}\right]+\nabla_{x} \Psi\right) \otimes\left(\mathbf{v}_{m}+\mathbf{V}\left[\mathbf{v}_{m}\right]+\nabla_{x} \Psi\right)}{h}-\mathbb{F}_{m}-\mathbb{M}\left[\mathbf{v}_{m}\right]\right] \\
<E-\delta_{m} \text { for some } \delta_{m} \searrow 0 .
\end{gathered}
$$

Now, fixing $m$ for a while, we apply Lemma 2.1 with

$$
N=2, U=(0, T) \times \Omega, r=h, \mathbf{g}=\mathbf{v}_{m}+\mathbf{V}\left[\mathbf{v}_{m}\right]+\nabla_{x} \Psi, \mathbb{W}=\mathbb{F}_{m}+\mathbb{M}\left[\mathbf{v}_{m}\right] \text {, and } e=E-\delta_{m} / 2 .
$$

Denoting $\left\{\left[\mathbf{w}_{m, n}, \mathbb{G}_{m, n}\right]\right\}_{n=1}^{\infty}$ the quantities resulting from the conclusion of Lemma [2.1, we consider

$$
\mathbf{v}_{m, n}=\mathbf{v}_{m}+\mathbf{w}_{m, n}, \mathbb{F}_{m, n}=\mathbb{F}_{m}+\mathbb{G}_{m, n}
$$

Obviously,

$$
\partial_{t} \mathbf{v}_{m, n}+\operatorname{div}_{x} \mathbb{F}_{m, n}=0, \operatorname{div}_{x} \mathbf{v}_{m, n}=0, \mathbf{v}_{m, n}(0, \cdot)=\mathbf{v}_{0},
$$

and, in accordance with Lemma 2.1,

$$
\lambda_{\max }\left[\frac{\left(\mathbf{v}_{m, n}+\mathbf{V}\left[\mathbf{v}_{m}\right]+\nabla_{x} \Psi\right) \otimes\left(\mathbf{v}_{m, n}+\mathbf{V}\left[\mathbf{v}_{m}\right]+\nabla_{x} \Psi\right)}{h}-\mathbb{F}_{m, n}-\mathbb{M}\left[\mathbf{v}_{m}\right]\right]<E-\delta_{m} / 2 .
$$

Consequently, in view of the continuity properties of the operators $\mathbf{v} \mapsto \mathbf{V}[\mathbf{v}], \mathbf{v} \mapsto \mathbb{M}[\mathbf{v}]$, we may conclude that for each $m$ there exists $n=n(m)$ such that

$$
\left[\mathbf{v}_{m, n(m)}, \mathbb{F}_{m, n(m)}\right] \in X_{0}, m=1,2, \ldots
$$

Moreover, in view of (2.24), we may suppose

$$
\mathbf{v}_{m, n(m)} \rightarrow \mathbf{v} \text { in } C_{\text {weak }}\left([0, T] ; L^{2}\left(\Omega ; R^{2}\right)\right) \text { as } m \rightarrow \infty,
$$

in particular,

$$
I\left[\mathbf{v}_{m, n}\right] \rightarrow I[\mathbf{v}] \text { as } m \rightarrow \infty .
$$

Since for each $m$

$$
\lim _{n \rightarrow \infty} \int_{0}^{T} \int_{\Omega} \frac{\left(\mathbf{v}_{m}+\mathbf{V}\left[\mathbf{v}_{m}+\mathbf{w}_{m, n}\right]+\nabla_{x} \Psi\right) \mathbf{w}_{m, n}}{h} \mathrm{~d} x \mathrm{~d} t=0,
$$


thus using once more Lemma 2.1 combined with Jensen's inequality, we observe that the sequence $\mathbf{v}_{m, n(m)}$ can be taken in such a way that

$$
\begin{gathered}
\liminf _{m \rightarrow \infty} I\left[\mathbf{v}_{m, n(m)}\right]=\liminf _{m \rightarrow \infty} \int_{0}^{T} \int_{\Omega}\left(\frac{1}{2} \frac{\left|\mathbf{v}_{m}+\mathbf{w}_{m, n(m)}+\mathbf{V}\left[\mathbf{v}_{m}+\mathbf{w}_{m, n(m)}\right]+\nabla_{x} \Psi\right|^{2}}{h}-E\right) \mathrm{d} x \mathrm{~d} t \\
=\lim _{m \rightarrow \infty} \int_{0}^{T} \int_{\Omega}\left(\frac{1}{2} \frac{\left|\mathbf{v}_{m}+\mathbf{V}\left[\mathbf{v}_{m}+\mathbf{w}_{m, n(m)}\right]+\nabla_{x} \Psi\right|^{2}}{h}-E\right) \mathrm{d} x \mathrm{~d} t+\liminf _{m \rightarrow \infty} \int_{0}^{T} \int_{\Omega} \frac{1}{2} \frac{\left|\mathbf{w}_{m, n(m)}\right|^{2}}{h} \mathrm{~d} x \mathrm{~d} t \\
\geq I[\mathbf{v}]+C_{1} \liminf _{m \rightarrow \infty} \int_{0}^{T} \int_{\Omega}\left(E-\delta_{m}-\frac{1}{2} \frac{\left|\mathbf{v}_{m}+\mathbf{V}\left[\mathbf{v}_{m}\right]+\nabla_{x} \Psi\right|^{2}}{h}\right)^{2} \mathrm{~d} x \mathrm{~d} t \\
\geq I[\mathbf{v}]+C_{2}(T,|\Omega|) \liminf _{m \rightarrow \infty}\left(\int_{0}^{T} \int_{\Omega}\left(E-\delta_{m}-\frac{1}{2} \frac{\left|\mathbf{v}_{m}+\mathbf{V}\left[\mathbf{v}_{m}\right]+\nabla_{x} \Psi\right|^{2}}{h}\right) \mathrm{d} x \mathrm{~d} t\right)^{2} \\
=I[\mathbf{v}]+C_{2}(T,|\Omega|)(I[\mathbf{v}])^{2}, C_{2}(T,|\Omega|)>0,
\end{gathered}
$$

which is compatible with (2.26) only if $I[\mathbf{v}]=0$.

Thus we have shown (2.25), and, consequently, Theorem 2.1.

\section{Dissipative solutions}

The solutions "constructed" in the proof of Theorem 2.1 satisfy (2.9), more specifically,

$$
\frac{1}{2} h|\mathbf{u}|^{2}=E=\Lambda-a h^{2}-\partial_{t} \Psi \text { for a.a. }(t, x) \in(0, T) \times \Omega .
$$

In particular, as $\Lambda$ has been chosen large, the total energy $E_{\text {tot }}$ of the flow,

$$
E_{\mathrm{tot}}(t)=\int_{\Omega}\left[\frac{1}{2} h|\mathbf{u}|^{2}+a h^{2}\right](t, \cdot) \mathrm{d} x
$$

may (and does in "most" cases) experience a jump at the initial time,

$$
\liminf _{t \rightarrow 0+} E_{\text {tot }}(t)>\int_{\Omega}\left[\frac{1}{2} h_{0}\left|\mathbf{u}_{0}\right|^{2}+a h_{0}^{2}\right] \mathrm{d} x .
$$

Apparently, solutions satisfying (3.1) are "non-physical" violating the First law of thermodynamics, at least if the forces $\mathbf{f}$ are regular. This observation leads to a natural admissibility criterion based on

the energy balance appended to the definition of weak solutions to eliminate the oscillatory solutions constructed in Theorem 2.1 . 


\subsection{Energy inequality and dissipative solutions}

For the sake of simplicity, suppose that $a>0$ is a positive constant independent of $x$. Taking, formally, the scalar product of equation (1.2) with $\mathbf{u}$ and integrating the resulting expression over $\Omega \times(0, \tau)$, we obtain the energy inequality

$$
\begin{aligned}
E_{\text {tot }}(\tau) \equiv \int_{\Omega}\left[\frac{1}{2} h|\mathbf{u}|^{2}+a h^{2}\right](\tau, \cdot) \mathrm{d} & +\int_{0}^{\tau} \int_{\Omega} h \gamma \mathbf{B}_{\mathbf{u}} \cdot \mathbf{u} \mathrm{d} x \mathrm{~d} t \leq \int_{\Omega}\left[\frac{1}{2} h_{0}\left|\mathbf{u}_{0}\right|^{2}+a h_{0}^{2}\right] \mathrm{d} x \\
& +\int_{0}^{\tau} \int_{\Omega} h \mathbf{f} \cdot \mathbf{u} \mathrm{d} x \mathrm{~d} t
\end{aligned}
$$

where the function $\mathbf{B}$ was introduced in (1.7).

We say that $\left[h, \mathbf{u}, \mathbf{B}_{\mathbf{u}}\right]$ is a dissipative weak solution to the Savage-Hunter system if, in addition to (1.5 1.7), the energy inequality (3.2) holds for a.a. $\tau \in(0, T)$.

\subsection{Relative energy and weak-strong uniqueness}

Our goal is to show that a dissipative and a strong solution emanating from the same initial data coincide as long as the latter exists. To this end, we revoke the method proposed by Dafermos [6] and later elaborated in [11, based on the concept of relative energy. We introduce the relative energy functional

$$
\mathcal{E}(h, \mathbf{u} \mid H, \mathbf{U})=\int_{\Omega}\left[\frac{1}{2} h|\mathbf{u}-\mathbf{U}|^{2}+P(h)-P^{\prime}(H)(h-H)-P(H)\right] \mathrm{d} x,
$$

where

$$
P(h)=a h^{2} .
$$

Now, exactly as in [11, Section 3], we may derive the relative energy inequality

$$
\begin{gathered}
\mathcal{E}(h, \mathbf{u} \mid H, \mathbf{U})(\tau)+\int_{0}^{\tau} \int_{\Omega} h \gamma \mathbf{B}_{\mathbf{u}} \cdot(\mathbf{u}-\mathbf{U}) \mathrm{d} x \mathrm{~d} t \leq \mathcal{E}\left(h_{0}, \mathbf{u}_{0} \mid H(0, \cdot), \mathbf{U}(0, \cdot)\right) \\
+\int_{0}^{\tau} \int_{\Omega} h\left(\partial_{t} \mathbf{U}+\mathbf{u} \nabla_{x} \mathbf{U}\right) \cdot(\mathbf{U}-\mathbf{u}) \mathrm{d} x \mathrm{~d} t+\int_{0}^{\tau} \int_{\Omega} h \mathbf{f} \cdot(\mathbf{u}-\mathbf{U}) \mathrm{d} x \mathrm{~d} t \\
+\int_{0}^{\tau} \int_{\Omega}\left((H-h) \partial_{t} P^{\prime}(H)+\nabla_{x} P^{\prime}(H) \cdot(H \mathbf{U}-h \mathbf{u})\right) \mathrm{d} x \mathrm{~d} t-\int_{0}^{\tau} \int_{\Omega} \operatorname{div}_{x} \mathbf{U}\left(a h^{2}-a H^{2}\right) \mathrm{d} x,
\end{gathered}
$$

where $\left[h, \mathbf{u}, \mathbf{B}_{\mathbf{u}}\right]$ is a dissipative weak solution of the Savage-Hutter system and $\left[H, \mathbf{U}, \mathbf{B}_{\mathbf{U}}\right]$ are sufficiently smooth functions in $[0, T] \times \Omega, H>0$.

We are ready to prove the following result: 
Theorem 3.1 Let $\left[h, \mathbf{u}, \mathbf{B}_{\mathbf{u}}\right]$ be a dissipative weak solution of the Savage-Hutter system in $(0, T) \times$ $\Omega$ in the sense specified through (1.5-1.7), (3.2). Let $\left[H, \mathbf{U}, \mathbf{B}_{\mathbf{U}}\right], H>0$ be a globally Lipschitz (strong) solution of the same problem, with

$$
h_{0}=H(0, \cdot), \mathbf{u}_{0}=\mathbf{U}(0, \cdot) .
$$

Then

$$
h=H, \mathbf{u}=\mathbf{U} \text { a.e. in }(0, T) \times \Omega .
$$

As $h, \mathbf{u}$ are uniquely determined, then from the balance of momentum one can recover $\mathbf{B}_{\mathbf{u}}$ such that $\mathbf{B}_{\mathbf{u}}=\mathbf{B}_{\mathbf{U}}$ for almost all $(t, x) \in(0, T) \times \Omega$.

Proof:

A simple density arguments shows that $[H, \mathbf{U}]$ may be used as test functions in the relative energy inequality (3.4). As the initial values coincide, the latter reads

$$
\begin{gathered}
\mathcal{E}(h, \mathbf{u} \mid H, \mathbf{U})(\tau)+\int_{0}^{\tau} \int_{\Omega} h \gamma \mathbf{B}_{\mathbf{u}} \cdot(\mathbf{u}-\mathbf{U}) \mathrm{d} x \mathrm{~d} t \\
\leq \int_{0}^{\tau} \int_{\Omega} h\left(\partial_{t} \mathbf{U}+\mathbf{u} \nabla_{x} \mathbf{U}\right) \cdot(\mathbf{U}-\mathbf{u}) \mathrm{d} x \mathrm{~d} t+\int_{0}^{\tau} \int_{\Omega} h \mathbf{f} \cdot(\mathbf{u}-\mathbf{U}) \mathrm{d} x \mathrm{~d} t \\
+2 a \int_{0}^{\tau} \int_{\Omega}\left((H-h) \partial_{t} H+\nabla_{x} H \cdot(H \mathbf{U}-h \mathbf{u})\right) \mathrm{d} x \mathrm{~d} t-\int_{0}^{\tau} \int_{\Omega} \operatorname{div}_{x} \mathbf{U}\left(a h^{2}-a H^{2}\right) \mathrm{d} x,
\end{gathered}
$$

where, furthermore,

$$
\begin{gathered}
h\left(\partial_{t} \mathbf{U}+\mathbf{u} \cdot \nabla_{x} \mathbf{U}\right) \cdot(\mathbf{U}-\mathbf{u}) \\
=h\left(\partial_{t} \mathbf{U}+\mathbf{U} \cdot \nabla_{x} \mathbf{U}\right) \cdot(\mathbf{U}-\mathbf{u})+h(\mathbf{u}-\mathbf{U}) \cdot \nabla_{x} \mathbf{U} \cdot(\mathbf{U}-\mathbf{u}) .
\end{gathered}
$$

As $\mathbf{U}$ is globally Lipschitz, the second term in (3.6) may be "absorbed" by the left-hand side of (3.5) via Gronwall's argument. Furthermore,

$$
\begin{aligned}
h\left(\partial_{t} \mathbf{U}\right. & \left.+\mathbf{U} \cdot \nabla_{x} \mathbf{U}\right) \cdot(\mathbf{U}-\mathbf{u})=\frac{h}{H}\left(\partial_{t}(H \mathbf{U})+\operatorname{div}_{x}(H \mathbf{U} \otimes \mathbf{U})\right) \cdot(\mathbf{U}-\mathbf{u}) \\
& =-h \gamma \mathbf{B}_{\mathbf{U}} \cdot(\mathbf{U}-\mathbf{u})-h \mathbf{f} \cdot(\mathbf{u}-\mathbf{U})-\frac{h}{H} \nabla_{x}\left(a H^{2}\right) \cdot(\mathbf{U}-\mathbf{u})
\end{aligned}
$$


Consequently, (3.5) reduces to

$$
\begin{gathered}
\mathcal{E}(h, \mathbf{u} \mid H, \mathbf{U})(\tau)+\int_{0}^{\tau} \int_{\Omega} h \gamma\left(\mathbf{B}_{\mathbf{u}}-\mathbf{B}_{\mathbf{U}}\right) \cdot(\mathbf{u}-\mathbf{U}) \mathrm{d} x \mathrm{~d} t \\
\leq c \int_{0}^{\tau} \mathcal{E}(h, \mathbf{u} \mid H, \mathbf{U})(t) \mathrm{d} t \\
+2 a \int_{0}^{\tau} \int_{\Omega}\left((H-h) \partial_{t} H+\nabla_{x} H \cdot(H \mathbf{U}-h \mathbf{U})\right) \mathrm{d} x \mathrm{~d} t-\int_{0}^{\tau} \int_{\Omega} \operatorname{div}_{x} \mathbf{U}\left(a h^{2}-a H^{2}\right) \mathrm{d} x .
\end{gathered}
$$

Finally, write

$$
\operatorname{div}_{x} \mathbf{U}\left(a h^{2}-a H^{2}\right)=\operatorname{div}_{x} \mathbf{U}\left(a h^{2}-2 a H(h-H)-a H^{2}\right)+2 a \operatorname{div}_{x} \mathbf{U} H(h-H),
$$

where, similarly to the above, the first term can be handled by Gronwall's argument. Seeing the $H$, $\mathrm{U}$ satisfy the equation of continuity (1.1) we conclude that

$$
\mathcal{E}(h, \mathbf{u} \mid H, \mathbf{U})(\tau)+\int_{0}^{\tau} \int_{\Omega} h \gamma\left(\mathbf{B}_{\mathbf{u}}-\mathbf{B}_{\mathbf{U}}\right) \cdot(\mathbf{u}-\mathbf{U}) \mathrm{d} x \mathrm{~d} t \leq c \int_{0}^{\tau} \mathcal{E}(h, \mathbf{u} \mid H, \mathbf{U})(t) \mathrm{d} t
$$

whence, by Gronwall's lemma, $\mathcal{E}(h, \mathbf{u} \mid H, \mathbf{U})(\tau)=0$ for a.a. $\tau \in(0, T)$.

Q.E.D.

\section{Concluding remarks}

In view of Theorem 3.1, we are tempted to say that imposing the energy inequality (3.2) eliminates the "non-physical" oscillatory solutions, the existence of which is claimed in Theorem 2.1. However, the method of convex integration may be used to obtain the following result that can be shown in the same way as [4, Theorem 4.2].

Claim. Under the hypotheses of Theorem 2.1, let $T>0, h_{0}$ be given. Then there exists

$$
\mathbf{u}_{0} \in L^{\infty}\left(\Omega ; R^{2}\right)
$$

such that the Savage-Hutter system admits infinitely many dissipative weak solutions in $(0, T) \times \Omega$ starting from the initial data $\left[h_{0}, \mathbf{u}_{0}\right]$.

We leave the proof of Claim to the interested reader.

Finally we would like to mention that the same results hold in case of different choice of friction term. According to [17] the Savage-Hutter model is well valid for sand avalanches, however for the case of snow avalanches there is often considered a second velocity-dependent contribution, e.g. $h|\mathbf{u}| \mathbf{u}$. In a consequence, in terms of new variables the friction term reads for some coefficients $\gamma_{1}, \gamma_{2} \geq 0$ as follows $-\left(\gamma_{1}\left(\frac{h}{2 E}\right)^{1 / 2}+\gamma_{2}\left(\frac{2 E}{h}\right)^{1 / 2}\right)\left(\mathbf{v}+\mathbf{V}+\nabla_{x} \Psi\right)$. 


\section{References}

[1] F. Bouchut, A. Mangeney-Castelnau, B. Perthame, J.-P. Vilotte. A new model of Saint Venant and Savage-Hutter type for gravity driven shallow water flows. C. R. Math. Acad. Sci. Paris 336(6):531-536, 2003.

[2] F. Bouchut, M. Westdickenberg. Gravity driven shallow water models for arbitrary topography. Commun. Math. Sci. 2(3):359-389, 2004.

[3] E. Chiodaroli. A counterexample to well-posedness of entropy solutions to the compressible Euler system. J. Hyperbolic Differ. Equ., 11(3):493-519, 2014.

[4] E. Chiodaroli, E. Feireisl, and O. Kreml. On the weak solutions to the equations of a compressible heat conducting gas. Ann. Inst. H. Poincaré Anal. Non Linéaire 32(1): 225-243, 2015.

[5] E. Chiodaroli, O. Kreml. On the energy dissipation rate of solutions to the compressible isentropic Euler system. Arch. Ration. Mech. Anal. 214(3): 1019-1049, 2014.

[6] C.M. Dafermos. The second law of thermodynamics and stability. Arch. Rational Mech. Anal., 70:167-179, 1979.

[7] C. De Lellis and L. Székelyhidi, Jr. On admissibility criteria for weak solutions of the Euler equations. Arch. Ration. Mech. Anal., 195(1):225-260, 2010.

[8] L. Desvillettes and C. Villani. On the trend to global equilibrium for spatially inhomogeneous kinetic systems: the Boltzmann equation. Invent. Math., 159(2):245-316, 2005.

[9] R.J. DiPerna, A.J. Majda, Oscillations and concentrations in weak solutions of the incompressible fluid equations. Comm. Math. Phys. 108(4): 667-689, 1987.

[10] D. Donatelli, E. Feireisl, and P. Marcati. Well/ill posedness for the Euler-Korteweg-Poisson system and related problems. Commun. Partial Differential Equations, 2014. To appear.

[11] E. Feireisl, Bum Ja Jin, and A. Novotný. Relative entropies, suitable weak solutions, and weakstrong uniqueness for the compressible Navier-Stokes system. J. Math. Fluid Mech., 14:712-730, 2012.

[12] E.D. Fernández-Nieto, F. Bouchut, D. Bresch, M.J. Castro Daz, A. Mangeney. A new SavageHutter type model for submarine avalanches and generated tsunami. J. Comput. Phys. 227(16): 7720-7754, 2008. 
[13] J. M. N. T. Gray and X. Cui. Weak, strong and detached oblique shocks in gravity-driven granular free-surface flows. J. Fluid Mech., 579:113-136, 2007.

[14] J. M. N. T. Gray, Y.-C. Tai, and S. Noelle. Shock waves, dead zones and particle-free regions in rapid granular free-surface flows. J. Fluid Mech., 491:161-181, 2003.

[15] P. Gwiazda. An existence result for a model of granular material with non-constant density. Asymptot. Anal., 30(1): 43-60, 2002.

[16] P. Gwiazda. On measure-valued solutions to a two-dimensional gravity-driven avalanche flow model. Math. Methods Appl. Sci. 28(18): 2201-2223, 2005.

[17] K. Hutter, Y. Wang, S. P. Pudasaini. The Savage-Hutter avalanche model: how far can it be pushed? Philos. Trans. R. Soc. Lond. Ser. A Math. Phys. Eng. Sci. 363(1832): 1507-1528, 2005 .

[18] C. Juez, J. Murillo, P. Garca-Navarro. 2D simulation of granular flow over irregular steep slopes using global and local coordinates. J. Comput. Phys. 255:166-204, 2013.

[19] M. Pelanti, F. Bouchut, A. Mangeney. A Roe-type scheme for two-phase shallow granular flows over variable topography. M2AN Math. Model. Numer. Anal. 42(5): 851-885, 2008.

[20] V. Scheffer. An inviscid flow with compact support in space-time. J. Geom. Anal. 3(4): 343-401, 1993.

[21] A. Shnirelman. On the nonuniqueness of weak solution of the Euler equation. Comm. Pure Appl. Math. 50(12): 1261-1286, 1997.

[22] E. Wiedemann Existence of weak solutions for the incompressible Euler equations. Ann. Inst. H. Poincaré Anal. Non Linéaire 28(5): 727-730, 2011.

[23] N. Zahibo, E. Pelinovsky, T. Talipova, and I. Nikolkin. Savage-Hutter model for avalanche dynamics in inclined channels: Analytical solutions. J. Geophys. Res., 115:B3402, 1-18, 2010. 